\title{
Association of E-cadherin, matrix metalloproteinases, and tissue inhibitors of metalloproteinases with the progression and metastasis of hepatocellular carcinoma
}

\author{
Zu-hua Gao ${ }^{1}$, Maria S Tretiakova², Wen-hua Liu $^{2}$, Can Gong², Peter D Farris ${ }^{1}$ and John Hart ${ }^{2}$ \\ ${ }^{1}$ Department of Pathology and Laboratory Medicine, University of Calgary and Calgary Laboratory Services, \\ Calgary, AB, Canada and ${ }^{2}$ Department of Pathology, Laboratory of Surgical Pathology, The University \\ of Chicago Hospitals, Chicago, IL, USA
}

\begin{abstract}
Molecular markers can provide additional information to traditional histomorphological evaluation for the assessment of tumor progression and predicting the likelihood of invasion and metastasis in various types of malignancies. We studied the association of E-cadherin, matrix metalloproteinases (MMPs), and tissue inhibitors of metalloproteinase with the progression and metastasis of hepatocellular carcinoma. Tissue microarray including six normal livers, 14 cirrhotic livers, 39 macroregenerative nodules, 16 dysplastic nodules, 22 grade I hepatocellular carcinomas, $\mathbf{4 3}$ grade II hepatocellular carcinomas, seven grade III hepatocellular carcinomas, and 10 metastatic hepatocellular carcinomas were stained immunohistochemically with antibodies against MMPs $-1,-2,-3,-7,-9$, tissue inhibitors of metalloproteinase-1, $-2,-3$, and E-cadherin. The intensities of staining were scored manually by two pathologists and verified by the Chromavision Automated Cellular Imaging System. Compared with normal liver, cirrhotic liver had significantly lower E-cadherin and tissue inhibitors of metalloproteinase-1 but higher MMP-1 and -7, which suggest a more favorable environment for tumor invasion and metastasis. Grade I and grade II hepatocellular carcinomas demonstrated high E-cadherin and decreased MMP-3 and -9, which may explain the rarity of extrahepatic metastasis in low-grade hepatocellular carcinomas despite the high circulatory volume of the liver. The histological progression from dysplastic nodule to well-differentiated hepatocellular carcinoma and to less differentiated tumors was associated with a gradual decrease in tissue expression of E-cadherin, tissue inhibitors of metalloproteinase-2 and -3. Metastatic hepatocellular carcinomas showed significantly lower level of tissue inhibitors of metalloproteinase-1, $-2,-3$ but higher level of MMP-7. These data suggest that tissue expression of E-cadherin, certain MMPs, and tissue inhibitors of metalloproteinases could be useful markers to predict the progression and metastasis of hepatocellular carcinoma.
\end{abstract}

Modern Pathology (2006) 19, 533-540. doi:10.1038/modpathol.3800554; published online 10 February 2006

Keywords: hepatocellular carcinoma; E-cadherin; matrix metalloproteinases; tissue inhibitor of metalloproteinases; immunohistochemistry

Hepatocellular carcinoma is the sixth most common cancer worldwide in terms of numbers of cases (626 000/year) but because of the very poor prognosis, it is the third most common cause of death from cancer (598 000/year). ${ }^{1}$ Intrahepatic metastasis and recurrence of the neoplasm after surgical removal remains high. ${ }^{2}$ However, extrahepatic

Correspondence: Dr Z-H Gao, MD, PhD, Department of Pathology and Laboratory Medicine, Foothills Medical Center, The University of Calgary, Room C1150E, 1403-29 Street NW, Calgary, AB, Canada T2N 2T9.

E-mail: zu-hua.gao@cls.ab.ca

Received 27 July 2005; revised and accepted 19 December 2005; published online 10 February 2006 metastasis is unusual considering the high proliferative activity of hepatocellular carcinoma and rich blood circulation of the liver. ${ }^{3,4}$ The molecular mechanisms that underline the clinical behavior of hepatocellular carcinoma are still poorly understood. Knowledge of the pathogenesis of tumor progression and metastasis of hepatocellular carcinoma could help us to predict the prognosis and to make decisions on adjuvant therapies for those patients.

Dispersion of tumor cells from the primary tumor is considered one of the key events for metastatic progression. Tumor cell dispersion relies on the loss of homotypic cell-cell adhesion, which is largely mediated by E-cadherin/catenin complex. ${ }^{5}$ 
E-cadherin, a transmembrane glycoprotein, mediates $\mathrm{Ca}^{2+}$-dependent cell-cell adhesion through its intracytoplasmic interaction with $\beta$ and $\alpha$ Catenin. $\alpha$-Catenin connects the cadherin-catenin complex to actin filament networks, leading to increased adhesive strength. Changes in adhesion complexes lead to alterations of cell polarity, proliferation, mobility, and differentiation. ${ }^{6}$ In a variety of cancers such as lobular breast carcinoma, diffuse gastric carcinoma, endometrial and ovarian carcinoma as well as hepatocellular carcinoma, reduced expression of E-cadherin because of genetic mutations, in combination with loss of heterozygosity at the E-cadherin gene ( $C D H 1)$, has been correlated with disruption of cell-cell contacts, epithelial-mesenchymal transition, invasiveness, and metastatic potential. ${ }^{7-11}$ However, this concept has been challenged in some studies of hepatocellular carcinoma. ${ }^{12-14}$

Invasion through basement membrane and interstitial extracellular matrix is another key event for metastatic progression, which requires the action of a series of proteolytic enzymes named matrix metalloproteinases (MMPs). ${ }^{15-17}$ MMPs are a group of zinc-dependent endopeptidases that share many structural and functional properties but with different substrate specificities. ${ }^{15,18,19}$ They are historically divided on the basis of their specificity for extracellular matrix components into collagenases (MMP-1, -2, -9), gelatinases (MMP-2, -3, -9), stromelysins (MMP-3, -10, -11), and matrilysin (MMP-7). ${ }^{20,21}$ As the list of MMP substrates grow (21 human MMPs have been identified thus far), they have been grouped into eight distinct structural classes, five are secreted and three are membranetype. $^{22}$ Enhanced MMP expressions have been reported in various human malignant tumors. ${ }^{16,17}$ Most clinical data show a correlation between MMP expression with advanced tumor stage, invasion, metastasis, and shortened survival. However, there are a few cases in which increased expression of specific MMPs reflects a favorable prognosis. ${ }^{23,24}$ The activity of MMPs is regulated by a group of molecules named tissue inhibitors of MMP (TIMPs) that reside in the normal tissue and counter react with MMPs with a 1:1 stoichiometry. ${ }^{15,19,20,25}$ There are four members of the TIMP family designated TIMP-1, -2 , -3 and -4 . TIMPs have been proposed to act selectively on different MMPs: TIMP-2, -1 and -3 preferentially bind to MMP-2, -9 and MMP-9/membrane type 1MMP, respectively. ${ }^{26}$ The recently cloned TIMP-4 has been shown to act on numerous MMPs. ${ }^{27}$

To gain insight into the involvement of MMPs, TIMPs, and E-cadherin in the progression and metastasis of hepatocellular carcinoma, we studied the expression patterns of E-cadherin, MMP-1, -2, -3, $-7,-9$, and TIMP-1, -2 and -3 in tissue samples of normal liver, cirrhotic liver, macroregenerative nodule, dysplastic nodule, hepatocellular carcinoma of various grade of differentiation, and metastatic hepatocellular carcinoma. The results elucidated the predictive value of immunohistochemical evaluation of MMPs, TIMPs, and E-cadherin expression for the progression and metastasis of hepatocellular carcinoma. The data also offered a possible explanation for the rarity of extrahepatic metastasis of low to moderate grade hepatocellular carcinoma and the effects of cirrhosis on tumor invasion and metastasis.

\section{Materials and methods}

\section{Tissue Samples}

With the approval of the institutional review board at the University of Chicago, we retrieved the following tissue samples from the Department of Pathology, University of Chicago Hospitals: six normal livers (two men and four women; age range 12-69 years), 14 cirrhotic livers (seven men and seven women; age range 46-90 years), 39 macroregenerative nodules (19 men and 20 women; age range 43-73 years), 16 dysplastic nodules (10 men and six women; age range $43-72$ years), 22 grade I hepatocellular carcinomas (14 men and eight women; age range 46-85 years), 43 grade II hepatocellular carcinomas (27 men and 16 women; age range 47-85 years), seven grade III hepatocellular carcinomas (two men and five women; age range 53-68 years), and 10 metastatic hepatocellular carcinomas (five men and five women; age range 18-80 years). The tissue samples of normal liver, cirrhotic liver, and hepatocellular carcinoma were initially obtained from resection specimens performed for metastatic tumors, explanted HCV-related cirrhotic livers, and surgically removed HCV-related hepatocellular carcinomas, respectively. The routine H\&E-stained tissue sections were reviewed by two pathologists and the tumors were graded using WHO grading system. ${ }^{28}$ The diagnostic criteria devised by the international working group were used for the selection of macroregenerative nodule and dysplastic nodule, with the latter one composed exclusively of small cell dysplasia. ${ }^{29}$ Representative areas were selected for the construction of the tissue microarray blocks using $1.5 \mathrm{~mm}$ punchers on the manual tissue arrayer MTA-1 (Beecher Instruments, Sun Prairie, WI, USA). Because heterogeneity of tumor differentiation is common, especially in large sized tumors, grading was based on the worst area and this area was selected when making the tissue microarray. Owing to the small number of cases with high-grade tumors, the grade III (poorly differentiated) and grade IV (undifferentiated) hepatocellular carcinomas were combined and designated as grade III in this study. All metastatic hepatocellular carcinomas were histologically high-grade tumors.

\section{Immunohistochemical Analysis}

Immunohistochemical staining was performed on $4 \mu \mathrm{m}$ sections obtained from formalin-fixed, 
Table 1 List of antibodies against MMPs, TIMPs, and E-cadherin

\begin{tabular}{|c|c|c|c|c|c|c|c|c|c|}
\hline & $M M P-1$ & $M M P-2$ & $M M P-3$ & $M M P-7$ & $M M P-9$ & E-cadherin & TIMP-1 & $T I M P-2$ & TIMP-3 \\
\hline Isotype & Poly & IgG1 & IgG2b & IgG2b & IgG1 & IgG1 & IgG1 & IgG2a & IgG2b \\
\hline Clone & - & $42-5 \mathrm{D} 11$ & SL-1 & ID2 & $23 \mathrm{C}$ & $4 \mathrm{~A} 2 \mathrm{C} 7$ & 2A5 & $3 \mathrm{~A} 4$ & $18 \mathrm{D} 12 \mathrm{~b}$ \\
\hline Species & Rabbit & Mouse & Mouse & Mouse & Mouse & mouse & mouse & mouse & mouse \\
\hline Dilution & $1: 750$ & $1: 100$ & $1: 20$ & $1: 25$ & $1: 30$ & $1: 100$ & $1: 20$ & $1: 20$ & $1: 50$ \\
\hline Source & Neomarkers & $\begin{array}{l}\text { Oncogene Research } \\
\text { Products }\end{array}$ & Neo-markers & Neo-markers & Novo-castra & Zymed & Novo-castra & Novo-castra & Novo-castra \\
\hline
\end{tabular}

paraffin-embedded tissue microarray blocks. After deparaffinization and rehydration, tissue sections were incubated with monoclonal antibodies against MMP-1, -2, -3, -7, -9, TIMP-1, -2, -3, and E-cadherin (Table 1). A subsequent reaction was performed with biotin-free HRP enzyme labeled polymer of EnVision plus detection system (DakoCytomation, Carpinteria, CA, USA). A positive reaction was visualized with diaminobenzidine solution followed by counterstaining with hematoxylin. Positive controls were selected according to the manufacturer's recommendations: squamous epithelium for E-cadherin; placenta, bladder, breast, and ovarian carcinomas for MMPs $-1,-2,-3-7$; macrophages in tonsil tissue for MMP-9 and TIMP-1; colonic adenocarcinoma for TIMP-2; and placenta for TIMP-3. Negative controls were prepared by using nonimmune mouse or rabbit IgGs. The intensity of membrane staining for E-cadherin and cytoplasmic staining for MMPs and TIMPs was graded blindly by two pathologists (ZG, WL) independently at different times using a 4-tiered $(0-3)$ grading system. Discrepancies in grading were resolved by simultaneous grading at a multihead microscope in the presence of a third pathologist (JH). The grading results were further verified by the automated Chromavision Cellular Imaging System (Clarient Inc., San Juan Capistrano, CA, USA).

\section{Statistical Analysis}

Nonparametric multiple analysis of variance was used to see the effect of a categorical variable (normal liver, cirrhotic liver, macroregenerative nodule, dysplastic nodule, etc) on multiple dependent variables (MMP-1, $-2,-3$, etc). The result shows that the effect can be regarded as significant $\left(\chi^{2}=294.02\right.$, $P<0.0001)$. Subsequently, a series of Kruskal-Wallis test on each dependent variables followed by Dunn's post hoc test were used to determine which tissue types differ significantly from others.

\section{Results}

The results are illustrated in Table 2, Figures 1-3.

Compared with normal liver, cirrhotic liver had significantly increased expression of MMP-1 $(P<0.001),-7 \quad(P<0.001)$ and decreased expression of E-cadherin $(P<0.05)$ and TIMP-1 $(P<0.05)$; macroregenerative nodule and dysplastic nodule had significantly increased expression of MMP-1 $(P<0.001)$; grade I hepatocellular carcinoma had significantly increased expression of MMP-7 $(P<0.01)$ and decreased expression of MMP-9 $(P<0.05)$; grade II hepatocellular carcinoma had significantly decreased expression of MMP-3 $(P<0.05)$ and $-9(P<0.01)$; metastatic hepatocellular carcinoma had significantly decreased expression of E-cadherin $(P<0.05)$, TIMP-1 $(P<0.01)$, $-2(P<0.05)$, $-3(P<0.05)$ but increased expression of MMP-7 $(P<0.001)$.

Compared with cirrhotic liver, macroregenerative nodule had significantly decreased expression of MMP-3 $(P<0.01)$ and -7 $(P<0.001)$; grade I hepatocellular carcinoma had significantly increased expression of E-cadherin $(P<0.01)$, but decreased expression of MMP-3 $(P<0.05)$; grade II hepatocellular carcinoma had significantly increased expression of E-cadherin $(P<0.01)$, but decreased expression of MMP-1 $(P<0.01),-3(P<0.001)$, and $-7 \quad(P<0.01)$; metastatic hepatocellular carcinoma had significantly decreased expression of MMP-1 $(P<0.001)$ and $-2(P<0.01)$.

Compared with macroregenerative nodule, grade I hepatocellular carcinoma had significantly decreased expression of MMP-1 $(P<0.05)$ and TIMP-3 $(P<0.01)$, but increased expression of MMP-7 $(P<0.01)$; grade II hepatocellular carcinoma had significantly decreased expression of MMP-1 $(P<0.001)$, -9 $(P<0.05)$, TIMP-2 $(P<0.05)$ and -3 $(P<0.001)$; grade III hepatocellular carcinoma had significantly decreased expression of MMP-1 $(P<0.05)$ and TIMP-2 $(P<0.05)$; metastatic hepatocellular carcinoma had significantly decreased expression of MMP-1 $(P<0.001),-2 \quad(P<0.01)$, TIMP-1 $(P<0.01),-3 \quad(P<0.001)$, but significantly increased expression of MMP-7 $(P<0.001)$.

Compared with dysplastic nodule, grade I hepatocellular carcinoma had significantly decreased expression TIMP-2 $(P<0.001)$ and $-3 \quad(P<0.01)$; grade II hepatocellular carcinoma had significantly decreased expression of MMP-1 $(P<0.01), \quad-3$ $(P<0.001)$, TIMP-2 $(P<0.001)$ and $-3 \quad(P<0.001)$; grade III hepatocellular carcinoma had significantly decreased expression of MMP-1 $(P<0.001)$ and TIMP-2 $(P<0.001)$; metastatic hepatocellular carcinoma had significantly decreased expression of MMP-2 $(P<0.01)$, TIMP-1 $(P<0.01),-2 \quad(P<0.001)$ and $-3(P<0.001)$. 
Table 2 Comparison of tissue expression of MMPs, TIMPs, and E-cadherin

\begin{tabular}{|c|c|c|c|c|c|c|c|c|c|}
\hline & $M M P-1$ & $M M P-2$ & $M M P-3$ & $M M P-7$ & $M M P-9$ & E-cadherin & TIMP-1 & TIMP-2 & TIMP-3 \\
\hline Normal:cirrhosis & $P<0.001$ & & & $P<0.001$ & & $P<0.05$ & $P<0.05$ & & \\
\hline Normal:MRN & $P<0.001$ & & & & & & & & \\
\hline Normal:DN & $P<0.001$ & & & & & & & & \\
\hline Normal:HCC-I & & & & $P<0.01$ & $P<0.05$ & & & & \\
\hline Normal:HCC-II & & & $P<0.05$ & & $P<0.01$ & & & & \\
\hline Normal:HCC-III & & & & & & & & & \\
\hline Normal:HCC-M & & & & $P<0.001$ & & $P<0.05$ & $P<0.01$ & $P<0.05$ & $P<0.05$ \\
\hline Cirrhosis:MRN & & & $P<0.01$ & $P<0.001$ & & & $P<0.05$ & & \\
\hline Cirrhosis:DN & & & & & & & & & \\
\hline Cirrhosis:HCC-I & & & $P<0.05$ & & & $P<0.01$ & & & \\
\hline Cirrhosis:HCC-II & $P<0.01$ & & $P<0.001$ & $P<0.01$ & & $P<0.01$ & & & \\
\hline Cirrhosis:HCC-III & & & & & & & & & \\
\hline $\begin{array}{l}\text { Cirrhosis:HCC-M } \\
\text { MRN:DN }\end{array}$ & $P<0.001$ & $P<0.01$ & & & & & & & \\
\hline MRN:HCC-I & $P<0.05$ & & & $P<0.01$ & & & & & $P<0.01$ \\
\hline MRN:HCC-II & $P<0.001$ & & & & $P<0.05$ & & & $P<0.05$ & $P<0.001$ \\
\hline MRN:HCC-III & $P<0.05$ & & & & & & & $P<0.05$ & \\
\hline MRN:HCC-M & $P<0.001$ & $P<0.01$ & & $P<0.001$ & & & $P<0.01$ & & $P<0.001$ \\
\hline DN:HCC-I & & & & & & & & $P<0.001$ & $P<0.01$ \\
\hline DN:HCC-II & $P<0.01$ & & $P<0.001$ & & & & & $P<0.001$ & $P<0.001$ \\
\hline DN:HCC-III & $P<0.001$ & & & & & & & $P<0.001$ & \\
\hline DN:HCC-M & & $P<0.01$ & & & & & $P<0.01$ & $P<0.001$ & $P<0.001$ \\
\hline HCC-I:II & & & & & & & & & \\
\hline HCC-I:III & & & & & & & & & \\
\hline HCC-I:M & & & & & & & & & \\
\hline HCC-II:III & & & & & & & & & \\
\hline HCC-II:M & & & & & & & & & \\
\hline HCC-III:M & & & & & & & & & \\
\hline
\end{tabular}

Blank space indicates no statistical significance. DN, dysplastic nodule; HCC, hepatocellular carcinoma; HCC-I (-II, -III), grade I (II, III) hepatocellular carcinoma; HCC-M, metastatic hepatocellular carcinoma; MRN, macroregenerative nodule.

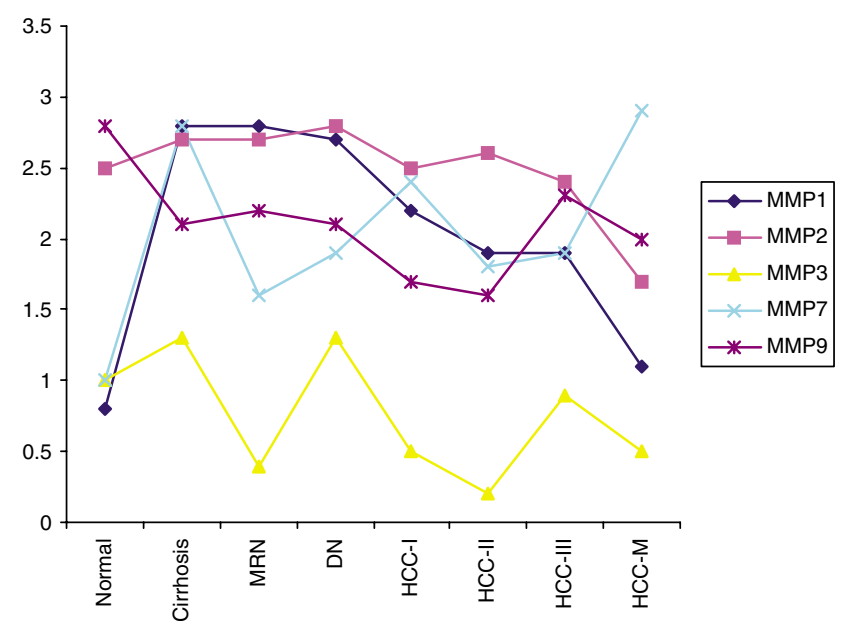

Figure 1 Expression of MMPs in different liver tissues. DN, dysplastic nodule; HCC, hepatocellular carcinoma; HCC-I (-II, -III), grade I (II, III) hepatocellular carcinoma; HCC-M, metastatic hepatocellular carcinoma; MRN, macroregenerative nodule.

There is no statistically significant difference in the tissue expression of MMPs, TIMPs, and E-cadherin between grade I and grade II hepatocellular carcinoma, grade I and grade III hepatocellular carcinoma, grade I and metastatic hepatocellular carcinoma, grade II and grade III hepatocellular carcinoma, grade III and metastatic hepatocellular carcinoma.

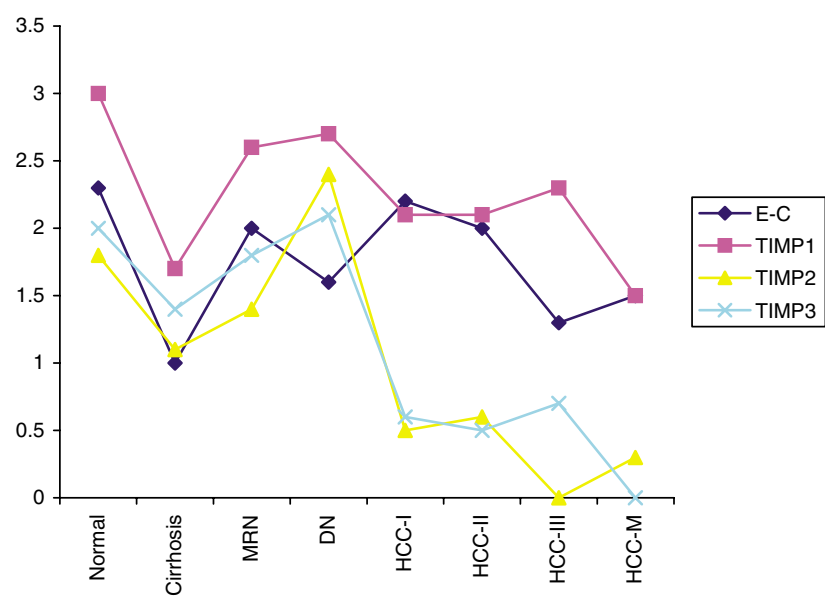

Figure 2 Expression of E-cadherin and tissue inhibitor of MMPs in different liver tissues. DN, dysplastic nodule; HCC, hepatocellular carcinoma; HCC-I (-II, -III), grade I (II, III) hepatocellular carcinoma; HCC-M, metastatic hepatocellular carcinoma; MRN, macroregenerative nodule.

From grade I, to grade II, to grade III and to metastatic hepatocellular carcinoma, there is progressive decrease of E-cadherin expression. From dysplastic nodule to grade I, to grade II, to grade III and to metastatic hepatocellular carcinoma, there is progressive decrease of TIMP expression, especially TIMP-2 and -3. 

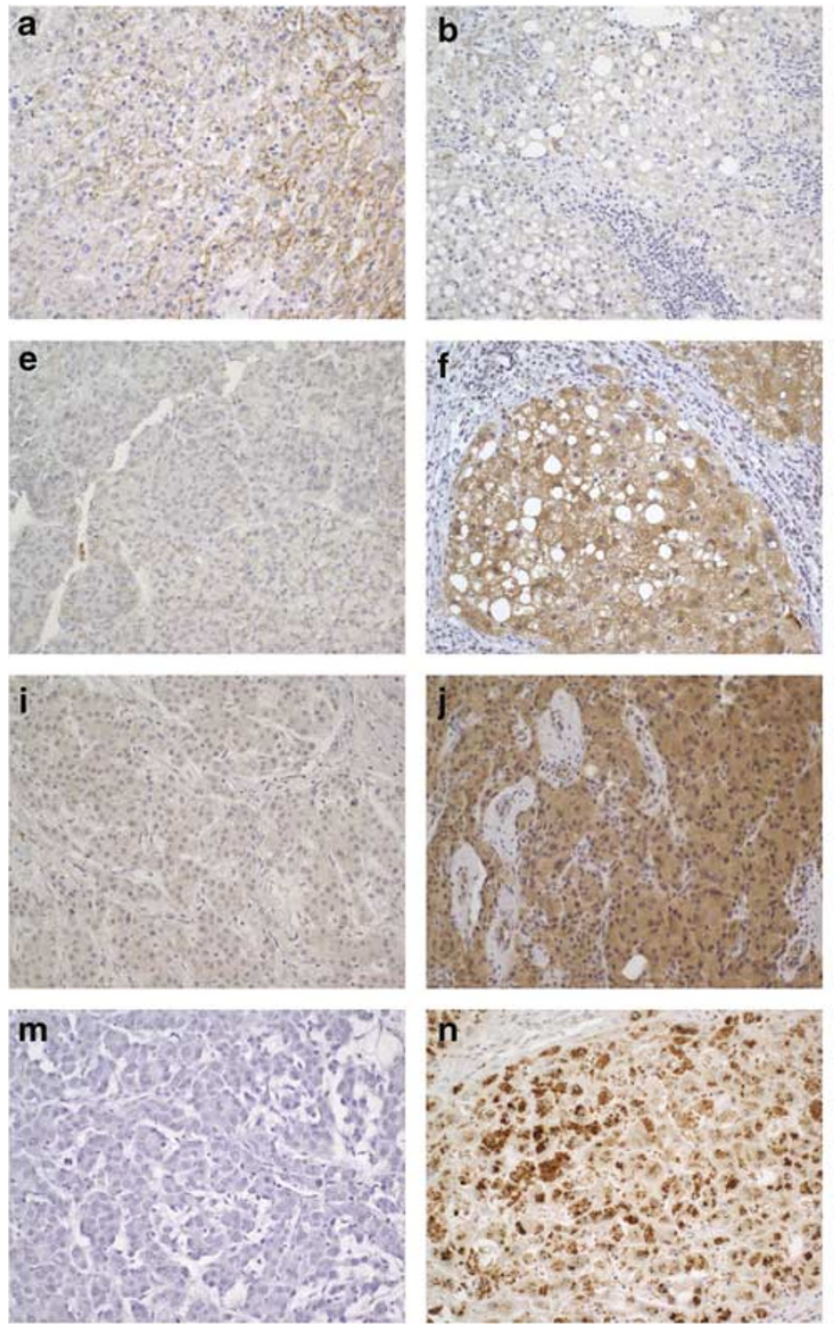
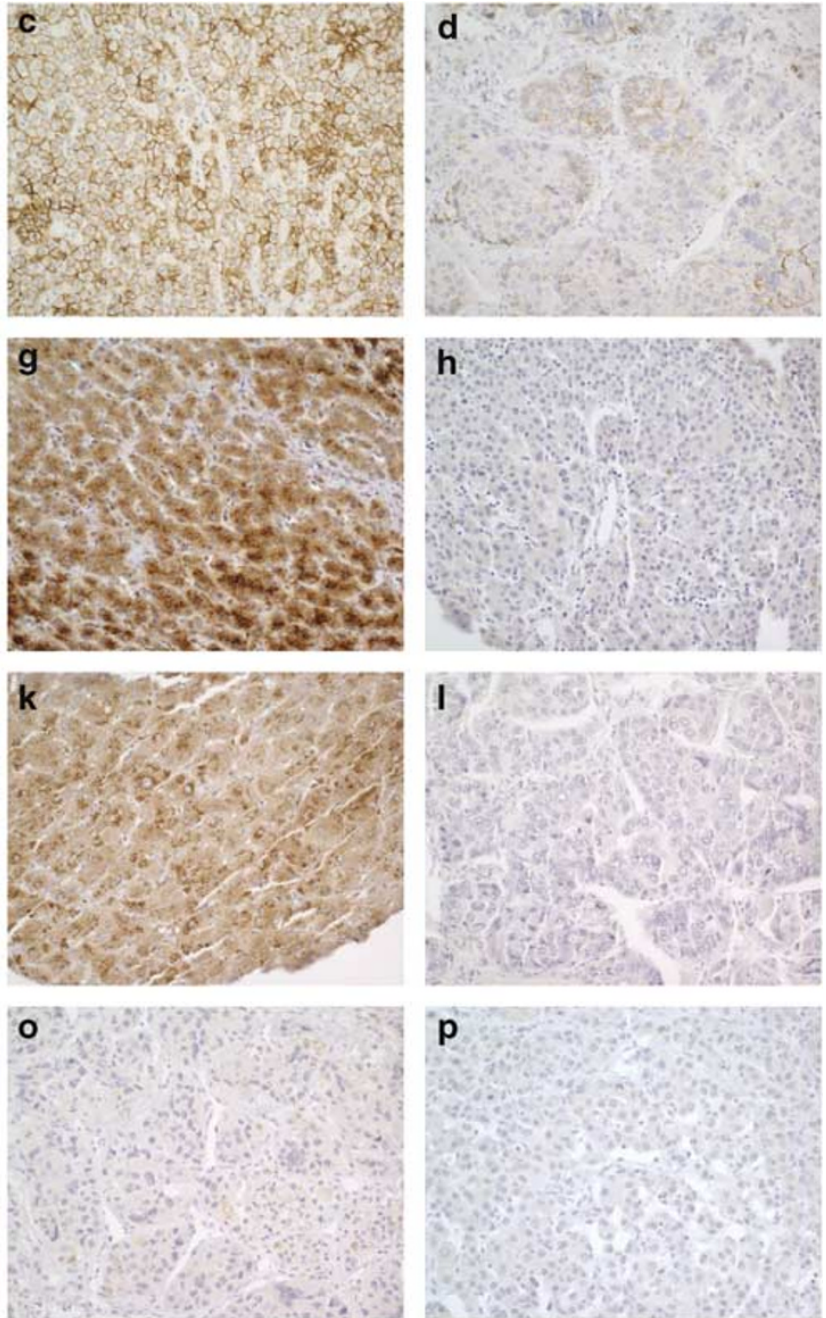

Figure 3 Representive expression patterns of E-cadherin, MMPs and TIMPs in different liver tissue (200 $\times$, immunoperoxidase). (a) E-cadherin expression in normal liver; (b) E-cadherin expression in cirrhotic liver; (c) E-cadherin expression in grade I hepatocellular carcinoma; (d) E-cadherin expression in grade III hepatocellular carcinoma; (e) E-cadherin expression in metastatic hepatocellular carcinoma; (f) MMP-1 expression in cirrhotic liver; (g) MMP-7 expression in cirrhotic liver; (h) MMP-3 expression in grade I hepatocellular carcinoma; (i) MMP-9 expression in grade I hepatocellular carcinoma; (j) MMP-7 expression in metastatic hepatocellular carcinoma; (k) TIMP-2 expression in dysplastic nodule; (l) TIMP-2 expression in grade III hepatocellular carcinoma; (m) TIMP-2 expression in metastatic hepatocellular carcinoma; (n) TIMP-3 expression in dysplastic nodule; (o) TIMP-3 expression in grade III hepatocellular carcinoma; (p) TIMP-3 expression in metastatic hepatocellular carcinoma.

\section{Discussion}

Hepatocellular carcinoma is multifactoral in etiology and complex in pathogenesis. The major risk factor is liver cirrhosis associated with chronic HBV and HCV infections, aflatoxin B exposure, and various metabolic disorders. ${ }^{30-32}$ Survival advantages of hepatocellular carcinomas arising from noncirrhotic liver over hepatocellular carcinomas arising from cirrhotic liver have been well documented..$^{33-35}$ This survival difference is believed to be because of the poor liver functional reserve in cirrhotic liver and the tendency towards development of a new primary after surgery. ${ }^{36,37}$ However, it is yet unknown whether there is any effect of cirrhosis on the metastatic potential of hepatocellular carcinomas. Significant increases of MMP-2,
MMP-9, TIMP-1 and TIMP-2 have been observed in an experimental hepatic fibrosis model. ${ }^{20,38,39}$ The difference in E-cadherin expression between cirrhotic liver and noncirrhotic normal liver has not been observed in previous studies. ${ }^{40}$ Our study demonstrated a significantly lower E-cadherin expression and higher expression of MMP-1, - 7 in cirrhotic liver tissue in comparison to noncirrhotic liver tissue. This observation suggests that cirrhosis provides a favorable environment for the invasion and intrahepatic metastasis of primary hepatocellular carcinoma. The balance between cirrhosis, in which abundant extracellular matrix is accumulated, and extracellular matrix degradation by factors secreted from tumor cells, might be one of the essential molecular process associated with the invasion and intrahepatic metastasis of hepatocellular carcinoma 
in cirrhotic liver. The finding of increased expression of MMP1 in macroregenerative nodule and dysplastic nodule in this study indicate common genetic alterations among cirrhotic liver, macroregenerative nodule, and dysplastic nodule.

Despite the high proliferative activity of hepatocellular carcinoma and rich blood circulation of the liver, extrahepatic metastasis is unusual ${ }^{3,4}$ especially for low-grade hepatocellular carcinomas. One of the proposed theories was that the increase in concentration of TIMP-1 in hepatocellular carcinoma cause increased type I collagen accumulation and consequent prevention of cellular detachment. ${ }^{3}$ In this study, we have found that expression of E-cadherin in grade I and grade II hepatocellular carcinomas is almost as high as normal liver. There was decreased expression of MMP-3 and -9 in both grade I and grade II hepatocellular carcinomas. The combinations of these findings provide, at least in part, an explanation for the rarity of extrahepatic metastasis of low-grade hepatocellular carcinoma. Liver transplantation might be able to cure those patients with well-differentiated hepatocellular carcinoma that have high E-cadherin expression and low level of MMP expression because of the low incidence of extrahepatic metastasis.

It has been well documented in several studies that as hepatocellular carcinoma progress from low to high histological grade, there is gradual loss of E-cadherin expression, which further correlates with vascular invasion and metastasis. ${ }^{41,42}$ In this study, hepatocytes in normal liver showed uniform high expression of E-cadherin. There is progressive decreased expression of E-cadherin from grade I through grade II and grade III to metastatic hepatocellular carcinoma. The expression of E-cadherin in metastatic hepatocellular carcinoma is statistically significantly lower than that of normal liver. These data reflect the importance of the adhesion junction system in the progression of hepatocellular carcinoma from low grade to high grade, and to metastatic carcinoma.

Studies about the association of MMPs with hepatocellular carcinoma have generally focused on the following three aspects: (1) those associated with carcinogenesis including overexpression of MMP-2 and MT1-MMP; (2) those associated with tumor progression including overexpression of MMP-2, -3, -9 and MT1-MMP; and (3) those associated with invasion and metastasis including overexpression of MMP-2, $-3,-9 .{ }^{43-47}$ In this study, we were unable to document progressive changes of MMPs as hepatocellular carcinoma progress from low to high grade except a significantly higher level of expression of MMP-7 in metastatic hepatocellular carcinoma. MMP-7, also know as matrilysin, is the smallest MMP. Overexpression of MMP-7 has been shown to be associated with metastatic progression of colorectal carcinoma and cholangiocarcinoma, as well as hepatocellular carcinoma. ${ }^{48-51}$ The observation of increased expression of MMP-7 in HCV- associated cirrhotic liver by us (see above) and others further emphasizes the role of this molecule in hepatocellular carcinoma carcinogenesis and metastatic progression. ${ }^{52}$

Overexpression of TIMP-1 but underexpression of TIMP-2 and -3 have been reported to be associated with invasion and metastasis in hepatocellular carcinoma. ${ }^{25,53,54}$ Early studies using recombinant TIMPs or basic gene transfer system (plasmids or retrovirus) have demonstrated that inhibition of MMPs by TIMPs blocks both tumor growth and local invasion. However, the use of TIMPs in clinical trials has proven largely disappointing. ${ }^{25,55}$ In this study, a low level of TIMP-1 expression was seen in cirrhotic liver tissue, but a high level of TIMP expression was observed in macroregenerative nodule (TIMP-1) and dysplastic nodule (TIMP-1, -2 and -3 ) tissue. However, the clinical implication of these observations is unclear. The progressive decrease of TIMP-2 and -3 tissue expression from dysplastic nodule to grade I, to grade II, to grade III and to metastatic hepatocellular carcinoma provides evidence for the involvement of TIMPs in hepatocellular carcinoma development and progression.

In summary, we have studied the tissue expression patterns of E-cadherin, MMP-1, -2, -3, -7, -9, and TIMP-1, -2 and -3 in tissue samples of normal liver, cirrhotic liver, macroregenerative nodule, dysplastic nodule, and hepatocellular carcinoma of various grade of differentiation. The increased expression of MMP-1, -7 and decreased expression of E-cadherin in cirrhotic liver suggests a more favorable environment for invasion and metastasis of hepatocellular carcinoma in comparison to noncirrhotic liver. Preserved E-cadherin and lower levels of MMP-3 and -9 may explain the rarity of extrahepatic metastasis in low-grade hepatocellular carcinoma despite the high circulatory volume of the liver. Decreased expression of E-cadherin, TIMP2, -3 and increased expression of MMP-7 could be useful markers for the prediction of tumor progression and metastasis. Once verified by larger scale studies, these observations are critical for the development of therapeutic strategies such as gene therapy to block tumor progression and to suppress invasion and metastasis.

\section{Acknowledgements}

We thank Dr Wanda Lester for assistance in editing the manuscript and Dr James Zhang for assistance in statistical analysis.

\section{References}

1 Parkin DM, Bray F, Ferlay J, et al. Global cancer statistics, 2002. CA Cancer J Clin 2005;55:74-108.

2 Niu Q, Tang ZY, Ma ZC, et al. Serum vascular endothelial growth factor is a potential biomarker 
of metastatic recurrence after curative resection of Hepatocellular carcinoma. World J Gastroenterol 2000; 6:565-568.

3 Akalin I, Güllü H, Kurdoglu M, et al. Why hepatocellular carcinoma cells are unlikely to metastasize: is there a role for tissue inhibitor of Metalloproteinase-1? Med Hypotheses 2001;57:221-223.

4 Olubuyide IO. The natural history of primary liver cell carcinoma: a study of 890 untreated adult Nigerians. Cent Afr J Med 1992;38:25-30.

5 Wijnhoven BPL, Dinjens WNM, Pignatelli M. Ecadherin-catenin cell-cell adhesion complex and human cancer. Br J Surg 2000;87:992-1005.

6 Kemler R. From cadherins to catenins: cytoplasmic protein interactions and regulation of cell adhesion. Trends Genet 1993;9:317-321.

7 Berx G, Cleton-Jansen AM, Noller F, et al. E-cadherin is a tumor/invasion suppressor gene mutated in human lobular breast cancers. EMBO J 1995;14:6107-6115.

8 Berx G, Becker KF, Hofler H, et al. Mutations of human E-cadherin (CDH1) gene. Hum Mutat 1998;12:226-237.

9 Inayoshi J, Ichida T, Sugitani S, et al. Gross appearance of hepatocellular carcinoma reflects E-cadherin expression and risk of early recurrence after surgical treatment. J Gastroenterol Hepatol 2003;18:673-677.

10 Saeki Y, Hazeki K, Matsumoto M, et al. Correlation between metastatic potency and the down-regulation of E-cadherin in the mouse hepatoma cell lines G-1 and G-5. Oncol Rep 2000;7:731-735.

11 Shinoyama Y, Hirohashi S. Cadherin intercellular adhesion molecule in hepatocellular carcinomas: loss of E-cadherin in an indifferentiated carcinoma. Cancer Lett 1991;57:131-135.

12 Osada T, Sakamoto M, Ino Y, et al. E-cadherin is involved in the intrahepatic metastasis of hepatocellular carcinoma. Hepatology 1996;24:1460-1467.

13 Wei Y, Tran Van Nhieu J, Prigent S, et al. Altered expression of E-cadherin in hepatocellular carcinoma: correlation with genetic alterations, $\beta$-catenin expression, and clinical features. Hepatology 2002;36:692-701.

14 Endo K, Ueda T, Ueyama J. Immunoreactive Ecadherin, alpha-catenin, beta-catenin, and r-catenin proteins in hepatocellular carcinoma: relationships with tumor grade, clinicopathologic parameters and patient survival. Hum Pathol 2000;31:558-565.

15 Brikedal-hansen H, Moore WGI, Bodden MK, et al. Matrix metalloproteinases: a review. Crit Rev Oral Biol Med 1993;4:197-250.

16 Yoshimoto M, Itoh F, Yamamoto H, et al. Expression of MMP-7 (Pump-1) mRNA in human colorectal cancers. Int J Cancer 1993;54:614-618.

17 Senota A, Itoh F, Yamamoto $\mathrm{H}$, et al. Relation of matrilysin messenger RNA expression with invasive activity in human gastric cancer. Clin Exp Metastasis 1998;16:313-321.

18 Docherty AJ, O’Conell J, Crabbe T, et al. The matrix metalloproteinases and their natural inhibitors: prospects for treating degenerative tissue diseases. Trends Biotechnol 1992;10:200-207.

19 Matrisian L. Metalloproteinases and their inhibitors in matrix remodeling. Trends Genet 1990;6:121-125.

20 Lichtinghagen R, Helmbrecht $\mathrm{T}$, Arndt $\mathrm{B}$, et al. Expression pattern of matrix metalloproteinases in human liver. Eur J Clin Chem Clin Biochem 1995;33: 65-71.

21 Kossakowska AE, Edwards DR, Lee SL, et al. Altered balance between matrix metalloproteinases and their inhibitors in experimental biliary fibrosis. Am J Pathol 1998;153:1895-1902.

22 Egeblad M, Werb Z. New functions for the matrix metalloproteinases in cancer progression. Nat Rev Cancer 2002;2:161-174.

23 Yang W, Arii S, Gorrin-Rivas MJ, et al. Human macrophage metalloelastase gene expression in colorectal carcinoma and its clinicopathologic significance. Cancer 2001;91:1277-1283.

24 Takeha S, Fujiyama Y, Bamba T, et al. Stromal expression of MMP-9 and urokinase receptor is inversely associated with liver metastasis and with infiltrating growth in human colorectal cancer: a novel approach from immune/inflammatory aspect. Jpn J Cancer Res 1997;88:72-81.

25 Baker AH, Edwards DR, Murphy G. Metalloproteinase inhibitors: biological actions and therapeutic opportunities. J Cell Sci 2002;115:3719-3727.

26 Curry Jr TE, Osteen KG. The matrix metalloproteinase system: changes, regulation, and impact through the ovarian and uterine reproductive cycle. Endocr Rev 2003;24:428-465.

27 Stratmann B, Farr M, Tschesche H. MMP-TIMP interaction depends on residue 2 in TIMP4. FEBS Lett 2001;507:285-287.

28 Hirohashi S, Ishak KG, Kojiro M, et al. Hepatocellular carcinoma. In: Hamilton SR and Aaltonen LA (eds). World Health Organization Classification of Tumors. Pathology and Genetics of Tumors of the Digestive System. IARC Press: Lyon, 2000, pp 165-166.

29 International Working Group. Terminology of nodular hepatocellular lesions. Hepatology 1995;22: 983-993.

30 Harris CC. Hepatocellular carcinogenesis: recent advances and speculations. Cancer Cells 1990;2:146-148.

31 Saito I, Miyanmura T, Ohabayashi A, et al. Hepatitis C virus infection is associated with the development of hepatocellular carcinoma. Proc Natl Acad Sci USA 1990;87:6547-6549.

32 Chen CJ, Wang LY, Lu SN, et al. Elevated aflatoxin exposure and increased risk of hepatocellular carcinoma. Hepatology 1996;24:38-42.

33 Nazeako UC, Goodman ZD, Ishak KG. Hepatocellular carcinoma in cirrhotic and noncirrhotic livers. A clinicohistopathologic study of 804 North American patients. Am J Clin Pathol 1996;105:65-75.

$34 \mathrm{Kew}$ MC. Hepatocellular carcinoma with and without cirrhosis. A comparison in southern African blacks. Gastroenterology 1989;97:136-139.

35 Okuda K, Nakashima T, Koijiro M, et al. Hepatocellular carcinoma without cirrhosis in Japanese patients. Gastroenterology 1989;97:140-146.

36 Kakar S, Burgart LJ, Batts KP, et al. Clinicopathologic features and survival in Fibrolamellar carcinoma: comparison with conventional hepatocellular carcinoma with and without cirrhosis. Mod Pathol 2005; 18:1417-1423.

37 Gao ZH. Predictive markers for the recurrence of hepatocellular carcinoma. J Surg Oncol 2005;92: 274-275.

38 Takahara T, Furui K, Funaki J, et al. Increased expression of matrix metalloproteinases-II in experimental liver fibrosis in rats. Hepatology 1995;21: 787-795.

39 Nie QH, Duan GR, Luo XD, et al. Expression of TIMP-1 and TIMP-2 in rats with hepatic fibrosis. World J Gastroenterol 2004;10:86-90. 
40 Ihara A, Koizumi H, Hashizume R, et al. Expression of epithelial cadherin and $\alpha$ - and $\beta$-Catenins in nontumoral livers and hepatocellular carcinomas. Hepatology 1996;23:1441-1447.

41 Matsumura T, Makino R, Mitamura K. Frequent downregulation of E-cadherin by genetic and epigenetic changes in malignant progression of hepatocellular carcinomas. Clin Cancer Res 2001;7:594-599.

42 Shimoyama Y, Hirohashi S. Caherin intercellular adhesion molecule in hepatocellular carcinomas: loss of E-cadherin in an undifferentiated carcinoma. Cancer Lett 1991;57:131-135.

43 Giannelli G, Bergamini C, Marinosci F, et al. Clinical role of MMP-2/TIMP-2 imbalance in hepatocellular carcinoma. Int J Cancer 2002;97:425-431.

44 Sakamoto Y, Mafune K, Mori M, et al. Overexpression of MMP-9 correlates with growth of small hepatocellular carcinoma. Int J Oncol 2000;17:237-243.

45 Arri S, Mise M, Harada T, et al. Overexpression of matrix metalloproteinase 9 gene in hepatocellular carcinoma with invasive potential. Hepatology 1996; 24:316-322.

46 Grigioni WF, Garisa S, D’Errico A, et al. Evaluation of hepatocellular carcinoma aggressiveness by a panel of extracellular matrix antigens. Am J Pathol 1991;138: 647-654.

47 Yamamoto $\mathrm{H}$, Itoh $\mathrm{F}$, Adachi $\mathrm{Y}$, et al. Relation of enhanced secretion of active matrix metalloproteinases with tumor spread in human hepatocellular carcinoma. Gastroenterology 1997;112:1290-1296.
48 Ishikawa T, Ichikawa Y, Mitsuhashi M, et al. Matrily$\sin$ is associated with progression of colorectal tumor. Cancer Lett 1996;107:5-10.

49 Adachi Y, Yamamoto $\mathrm{H}$, Itoch $\mathrm{F}$, et al. Contribution of matrilysin (MMP-7) to the metastatic pathway of human colorectal cancers. Gut 1999;45:252-258.

50 Miwa S, Miyagawa S, Soeda J, et al. Matrix metalloproteinase-7 expression and biologic aggressiveness of cholangiocellular carcinoma. Cancer 2002;94: 428-434.

51 Ishii Y, Nakasato Y, Kobayashi S, et al. A study on angiogenesis-related matrix metalloproteinase networks in primary hepatocellular carcinoma. J Exp Clin Cancer Res 2003;22:461-470.

52 Lichtinghagen R, Michels D, Haberkorn CI, et al. Matrix metalloproteinase (MMP)-2, MMP-7, and tissue inhibitor of metalloproteinase-1 are closely related to the fibroproliferative process in the liver during chronic hepatitis C. J Hepatol 2001;34:239-247.

$53 \mathrm{Lu}$ GL, Wen JM, Xu JM, et al. Relationship between TIMP-3 expression and promoter methylation of TIMP3 gene in hepatocellular carcinoma. Zhonghua Bing Li Xue Za Zhi 2003;32:230-233.

54 Nakatsukasa H, Ashida K, Higashi T, et al. Cellular distribution of transcripts for tissue inhibitor of metalloproteinases 1 and 2 in human hepatocellular carcinomas. Hepatology 1996;24:82-88.

55 Coussens LM, Fingleton B, Matrisian LM. Matrix metalloproteinase inhibitors and cancer: trials and tribulations. Science 2002;295:2387-2392. 